\title{
Indicadores de gestión en los procesos de admisión estudiantil en las universidades públicas venezolanas
}

\author{
Management indicators in the student admission processes in the venezuelan \\ public universities
}

\begin{tabular}{l|r|}
\cline { 2 - 2 } & Anargelis Chacín \\
\hline $\begin{array}{l}\text { Artículo recibido en mayo 2020 } \\
\text { Arbitrado en junio 2020 } \\
\text { Publicado en septiembre 2020 }\end{array}$ & anargelisch@gmail.com \\
& ORCID: 0000-0003-0012-2924 \\
Universidad del Zulia, Venezuela
\end{tabular}

RESUMEN | El actual estudio tuvo como propósito analizar los indicadores de gestión en los procesos de admisión estudiantil en las universidades públicas venezolanas. Metodológicamente se tipificó como descriptiva, con un diseño no experimental, transeccional y de campo. La población estuvo constituida por el personal encargado del proceso de admisión estudiantil de las universidades públicas venezolanas, ubicadas en la Costa Oriental del Lago. Para la recolección de datos se utilizó la encuesta a través un cuestionario; el cual quedó conformado por 15 ítems con escala de frecuencia, se utilizó el juicio de expertos para la validez del mismo. La confiabilidad fue calculada por el método Alfa de Cronbach, cuyo resultando fue 0,95. El análisis de los datos se realizó mediante estadística descriptiva, a través del estudio de la media aritmética. Los resultados arrojan alta presencia de los indicadores de gestión: efectividad, eficacia y eficiencia.

Palabras clave:

Efectividad, eficacia, eficiencia, indicadores de gestión, procesos de admisión estudiantil.

\begin{tabular}{l|l} 
ABSTRACT & $\begin{array}{l}\text { The purpose of the current study was to analyze the management indicators in the student } \\
\text { admission processes in the Venezuelan public universities. Methodologically it was typified as } \\
\text { descriptive, with a non-experimental, transectional and field design. The population was } \\
\text { made up of the personnel in charge of the student admission process of the Venezuelan } \\
\text { public universities, located on the East Coast of the Lake. For data collection, the survey was } \\
\text { used through a questionnaire; which was made up of } 15 \text { items with frequency scale, expert } \\
\text { judgment was used for its validity. Reliability was calculated by Cronbach's Alpha method, } \\
\text { the result of which was } 0.95 \text {. The data analysis was performed using descriptive statistics, } \\
\text { through the study of the arithmetic mean. The results show a high presence of management } \\
\text { indicators: effectiveness, efficacy and efficiency. }\end{array}$
\end{tabular}

Keywords: Effectiveness, efficiency, efficiency, management indicators, student admission processes 


\section{INTRODUCCIÓN}

Ante los rápidos cambios y los evidentes resultados del fenómeno de la globalización, las universidades se han visto en la necesidad de adecuar sus procesos para adaptarse a los nuevos eventos del contexto, y así cumplir con su misión. Ello debe ocasionar su transformación en organizaciones que innovan y aprenden de su propia experiencia y desempeño, para lograr su permanencia en la sociedad mediante una gestión fundamentada en la excelencia.

En este sentido, las universidades han transitado un largo camino desde su creación, ofreciendo 0 al menos intentando, dar respuestas a las demandas del contexto histórico, político, económico y social a través del cual han evolucionado. Visto así, es función de estas instituciones ofrecerle al estudiantado oportunidades para que defina su campo de estudio y trabajo, brindarle una capacitación científica, humanística y técnica que le permita incorporarse al trabajo productivo y orientarlo para la prosecución de estudios de educación superior.

De tal manera, en Venezuela las universidades están impregnadas de una gestión que requiere de una planificación unida a los indicadores dentro de cada institución, propiciando ventajas para el desarrollo de las acciones administrativas y académicas que en el sector educativo soportan el servicio que se presta, siendo uno de ellos los procesos de admisión estudiantil, que según Blanco, Flórez y Giménez. (2010) son un conjunto de procedimientos o medios que diseñan y utilizan las instituciones universitarias para captar estudiantes que en sus estudios universitarios obtengan un rendimiento adecuado.
En el caso específico de las universidades públicas de la Costa Oriental del Lago, el proceso de admisión se centra en los fundamentos legales de la educación universitaria venezolana, su artículo 3 indica la finalidad de la educación, así también, desde el 4 al 9 van señalando los derechos, vinculados del proceso educativo cuyo fin principal es la inserción de jóvenes según sea el plan de crecimiento matricular de cada institución.

El mismo, debe ser garante de la captación de nuevos ingresos a través de la promoción de los programas académicos, relacionándose éstos con los procesos de inscripción y todos los movimientos de permanencia, registro y control de la data académica de la población estudiantil de pregrado; de este modo cumplir con los procesos de culminación de estudios de los estudiantes que conlleven al solemne acto de grado, expedición de certificados, solvencias y solicitudes académicas.

En este orden de ideas, parte de la estrategia de las instituciones universitarias es lograr medir cada uno de los objetivos planteados en su estrategia organizacional, para ello, normalmente se hace uso de los indicadores de gestión, que según Pérez (2009, p. 1) se definen como "la expresión cuantitativa del comportamiento y desempeño de un proceso". Asimismo, Rodríguez y Gómez (2008, p.104), definen los indicadores de gestión como "Expresiones cuantitativas a través de las cuales se busca analizar la gestión de una unidad orgánica, en áreas como el uso de recursos (eficiencia) o, cumplimiento de programas (efectividad).

Por lo tanto, los indicadores de gestión son incluidos en los planes estratégicos con el fin de detectar el nivel de adecuación entre las metas y 
objetivos establecidos y los logros que se van alcanzando, de manera que permitan revelar la necesidad de eliminar o reformular aquellas acciones no conducentes a los objetivos perseguidos con suficiente antelación, para evitar situaciones no deseadas.

Es importante mencionar que la ventaja fundamental del uso de indicadores de gestión es la reducción drástica de la incertidumbre, la angustia y la subjetividad, obteniendo como resultado el incremento de la efectividad de la organización, así como también el bienestar de todos los trabajadores. Según Serna (2008), un indicador de gestión se define como una relación entre variables que permite observar aspectos de una situación y compararlos con las metas y objetivos propuestos. Dicha comparación permite observar la situación y las tendencias de evolución de la situación o fenómeno observado.

Sin embargo, y aun cuando en las universidades públicas de la Costa Oriental del Lago se ha venido observando un mayor reconocimiento de la importancia que tiene lograr una efectiva gestión en los procesos de admisión estudiantil, según la percepción del personal que labora en la misma y referido a la investigadora a través de una entrevista no estructurado, existen deficiencias al ofrecer este servicio con la calidad esperada, reconocido a través de los indicadores de gestión que les informan, si las metas y objetivos establecidos se están cumpliendo con eficacia.

En tal sentido, a criterio de la investigadora, un buen sistema de indicadores de medición de la gestión contribuye a poner en evidencia, en forma sistemática y rigurosa, las debilidades que caracterizan a ésta y todas las universidades venezolanas en términos del cumplimiento de sus funciones $y$, por consiguiente, proponer alternativas para mejorar los sistemas y procedimientos de gestión y como consecuencia, la calidad de los procesos y productos que generan estas instituciones. Finalmente, existen diversas maneras mediante las cuales un sistema de indicadores puede propiciar cambios que afecten positivamente el funcionamiento y calidad institucional.

En razón de ello, se presenta esta investigación cuyo objetivo se centra en analizar los indicadores de gestión en los procesos de admisión estudiantil en las universidades públicas venezolanas, a fin de generar para cada una de las funciones estratégicas de la política de admisión estudiantil un sentido en la medida en que sean concebidos y utilizados no como instrumentos estáticos e inflexibles con el único propósito de suministrar una visión transversal de la situación que intentan reflejar, sino como medios susceptibles de ser actualizados y modificados en forma permanente de acuerdo con los cambios que se consideren pertinentes.

\section{Indicadores de gestión}

Establece Pardo y Rodríguez (2007), que los indicadores de gestión, son aquellos que entregan información del desempeño desde el punto de vista de la actuación en la generación de productos y aquellos que se elaboran desde el punto de vista del desempeño de dichas actuaciones en las dimensiones, como lo son los de eficiencia, eficacia, calidad y economía.

Por su parte, Rincón (2010), señala que un indicador de gestión es aquel dato que refleja cuáles fueron las consecuencias de acciones tomadas en el pasado en el marco de una organización. Los expertos suelen definir muchos tipos de indicadores; entre los más frecuentes se encuentran los siguientes: en base a sus dimensiones, se habla de indicadores 
económicos, de efectividad y de eficiencia; desde un punto de vista clásico, existen los de aumento de ventas, los de disminución de costos, de la misma manera, es habitual que en la actualidad se recurra al uso de estos seis indicadores fundamentales: los de ventaja competitiva, los de flexibilidad, los de calidad de servicio, los de desempeño financiero, los de uso de recursos y los que vienen a dejar patente la innovación, entre otros.

Del mismo modo, Amat (2010), opina que en el desarrollo de los indicadores se deben identificar necesidades propias del área involucrada, clasificando según la naturaleza de los datos y la necesidad del indicador. Los indicadores se pueden clasificar en indicadores de eficacia o de eficiencia. El indicador de eficacia mide el logro de los resultados propuestos. Indica si se hicieron las cosas que se debían hacer, los aspectos correctos del proceso, mientras que el de eficiencia miden el nivel de ejecución del proceso, se concentran en el cómo se hicieron las cosas y miden el rendimiento de los recursos utilizados por un proceso.

En el contexto de orientación hacia los procesos, afirma Serna (2008), un medidor o indicador puede ser de proceso o de resultados. En el primer caso, se pretende medir que está sucediendo con las actividades, y en segundo se quiere medir las salidas del proceso. También se pueden clasificar los indicadores, según lo plantea este autor en indicadores de eficacia, eficiencia y efectividad; los cuales pueden ser utilizados para medir el desempeño de cada una de las áreas funcionales de la empresa (calidad, productividad, finanzas, mercadeo, operaciones, producción, ventas, atención al cliente, entre otros).
Así pues, según Serna (2008), el indicador de eficacia mide el logro de los resultados propuestos, indica si se hicieron las cosas que se debían hacer, los aspectos correctos del proceso. Se enfocan en el qué se debe hacer, por tal motivo, en el establecimiento de un indicador de eficacia es fundamental conocer y definir operacionalmente los requerimientos del cliente del proceso para comparar lo que entrega el proceso contra lo que él espera. De lo contrario, se puede estar logrando una gran eficiencia en aspectos no relevantes para el cliente.

Los indicadores de eficiencia miden el nivel de ejecución del proceso, se concentran en el cómo se hicieron las cosas y miden el rendimiento de los recursos utilizados por un proceso. Tienen que ver con la productividad. Los indicadores de efectividad involucran la eficiencia y la eficacia, es decir el logro de los resultados programados en el tiempo y con los costos más razonables posibles

Con referencia a los conceptos emitidos por los autores, la investigadora deduce que, los tipos de indicadores de gestión permiten medir con parámetros enfocados a la toma de decisiones, son señales para monitorear la gestión, asegurando que las actividades vayan en el sentido correcto y permitan evaluar los resultados de una gestión frente a sus objetivos, metas y responsabilidades. A fines del trabajo, se consideró importante ajustar o administrar el conjunto de indicadores a cada proceso, alineándolo con los de sus respectivas unidades de negocio y por tanto con la misión de la organización, para lograr la efectividad de los objetivos estratégicos propuestos; de allí que los tipos de indicadores de gestión que se identifican en esta investigación, acorde a Serna 
(2008), son los de: eficacia, eficiencia y efectividad.

\section{Indicadores de eficacia}

Apunta Pardo y Rodríguez (2007), que el indicador de eficacia mide el logro de los resultados propuestos. Indica si se hicieron las cosas que se debían hacer, los aspectos correctos del proceso. Los indicadores de eficacia se enfocan en el qué se debe hacer, por tal motivo, en el establecimiento de un indicador de eficacia es fundamental conocer y definir operacionalmente los requerimientos del cliente del proceso para comparar lo que entrega el proceso contra lo que él espera. De lo contrario, se puede estar logrando una gran eficiencia en aspectos no relevantes para el cliente.

Continuando, Pérez (2009) establece que los indicadores de eficacia llevan de forma inherente la definición previa de objetivos y el seguimiento de éstos a través de un sistema mínimo de información que permita informar sobre aspectos básicos del programa o la gestión a ser evaluada, entre los que se mencionan: productos que entrega el programa o el senvicio, usuarios a quienes de dirige (número, características), objetivos principales o estratégicos (logro que se pretende) obtener, mejorar, ampliar, optimizar) y metas concretas con las cuales hacer el seguimiento (cuándo, dónde, en qué condiciones), entre otros.

Ahora bien, según Rodríguez (2013), la eficacia es el grado en que una actividad, programa u organización alcanza sus metas y sus objetivos, medido a partir de sus productos (cantidad y calidad esperados, y efectos inmediatos). La eficacia demuestra la capacidad de una empresa u organización de cualquier tipo para desarrollar y mantener sistemáticamente unas ventajas comparativas que le permitan disfrutar y sostener una posición destacada en el entorno socio económico en que actúa.

Con base a lo descrito, la investigadora infiere que, el indicador eficacia se refiere a los resultados en relación con las metas y cumplimiento de los objetivos organizacionales. Para ser eficaz se deben priorizar las tareas y realizar ordenadamente aquellas que permiten alcanzarlos mejor y más rápidamente. En conclusión, la eficacia es la capacidad de escoger los objetivos apropiados para que el gerente sea capaz de seleccionar los objetivos correctos para trabajar en el sentido de alcanzarlos.

\section{Indicadores de eficiencia}

Señalan Pacheco, Castañeda y Caicedo, (2002), que este puede ser definido como el elemento de estadística que permite identificar la relación que existe entre las metas alcanzadas, tiempo y recursos consumidos con respecto a un estándar, una norma o una situación semejante. El mismo hace posible dimensionar el logro del máximo de resultados con el mínimo de recursos utilizados. En otras palabras, miden el nivel de ejecución del proceso, se concentran en el cómo se hicieron las cosas y miden el rendimiento de los recursos utilizados por un proceso.

Mientras, Rodríguez (2013), la define como la virtud y facultad para lograr un efecto determinado. El mismo autor expresa que, elaborar la eficiencia de la producción significa alcanzar mayores resultados económicos con los menores gastos de trabajo, siendo elementos fundamentales para su elaboración. Afirma que, la eficiencia es la capacidad para lograr un fin empleando los mejores medios posibles. 
Estos indicadores, en opinión de Bahamón (2010) permiten evaluar el grado de consecución de los objetivos estratégicos. Su utilidad depende de que, a la hora de proceder a su definición y establecimiento se garantice su fiabilidad y consistencia, evitando ambigüedades. Hay que tener en cuenta que su objetivo último es facilitar el análisis de la situación para dotar de precisión y eficacia a la toma de decisiones, desde un conocimiento óptimo de la realidad de la empresa.

Bajo esta perspectiva, se acota lo señalado por Corredor (2007), para quien la eficiencia es la maximización de los insumos empleados para generar productos o servicios; ya sea que con recursos iguales o constantes se obtengan mayores resultados $o$ que con resultados iguales o constantes se ejecuten menores recursos. Mide la manera en que los recursos e insumos se convierten económicamente en resultados o productos.

Para este autor, algunos de los objetivos de los indicadores que miden el factor de eficiencia son: medir la productividad de los procesos en relación al recurso utilizado frente al servicio prestado o la actividad desarrollada; establecer el nivel óptimo en la utilización de los recursos para el logro de objetivos previstos; determinar si la cobertura alcanzada en la elaboración del producto o prestación del servicio alcanzó las metas establecidas al mínimo costo.

Frente a los postulados expuestos, la investigadora infiere que, la eficiencia es la capacidad administrativa de producir el máximo de resultados con el mínimo de recursos, el mínimo de energía y en el mínimo de tiempo posible; teniendo en cuenta que eficaz tiene que ver con hacer efectivo un intento o propósito. Los indicadores de eficiencia se emplean para relacionar los esfuerzos frente a los resultados que se obtengan; a mayores resultados, mayor eficiencia. Si se obtiene mejores resultados con menor gasto de recursos o menores esfuerzos, se habrá incrementado la eficiencia.

\section{Indicadores de efectividad}

Según Barbosa (2010), los indicadores de efectividad se enfocan en el impacto producido por los productos y/o servicios sobre los clientes y usuarios. Se relacionan, con la medición del nivel de satisfacción del usuario, que aspira a recibir un producto o servicio en condiciones favorables de costo y oportunidad, así como establecer el grado de cobertura del servicio prestado. En conclusión, la efectividad está relacionada con las respuestas que demos al interrogante ¿Para qué se hizo? Este tipo de indicadores mide el impacto final alcanzado frente a los bienes o servicios generados sobre los clientes y usuarios.

En igual forma, Pérez y Magro (2011) establecen que los indicadores de efectividad constituyen una herramienta para medir el cumplimiento de los objetivos de cualquier proceso o institución. Lo que no se mide no se puede controlar, y lo que no se controla no se puede gestionar. No se pueden tomar decisiones por simple intuición. Estos mostrarán los puntos problemáticos del proceso y ayudarán a caracterizarlos, comprenderlos y confirmarlos. El desarrollo de una tecnología de medición de la efectividad de la gestión cae indefectiblemente en esta arista de la actividad humana, la administración. Debería ser en los gerentes donde surgiera la necesidad de medir el desempeño de su organización en relación al mercado y los competidores, y a su vez quienes primero construyeran las propuestas tecnológicas que más se adecuen al contexto organizacional. 
Ahora bien, para Jaramillo (2011), los indicadores de efectividad involucran eficiencia y eficacia, es decir, logro de los resultados programados en el tiempo con los costos más razonables posibles, supone hacer lo correcto con exactitud y sin ningún desperdicio de tiempo o dinero. Agrega Rodríguez (2013), la efectividad es la relación entre lo producido y la necesidad del servicio o del producto, es decir mide en qué grado la actividad cumplida satisface la demanda del bien o servicio en términos generales.

De acuerdo a lo emitido por los autores, para la investigadora la efectividad significa cuantificación del logro de la meta, también como la capacidad de lograr el efecto que se desea. Los indicadores de efectividad, tienen que ver con hacer realidad un intento o propósito, y están relacionados con el cumplimiento al ciento por ciento de los objetivos planteados, teniendo en cuenta que eficiencia tiene que ver con la actitud y la capacidad para llevar a cabo un trabajo o una tarea con el mínimo gasto de tiempo. Se le considera como una relación entre los resultados logrados y los propuestos, o sea permite medir el grado de cumplimiento de los objetivos planificados.

\section{MÉTODO}

La investigación se tipificó como descriptiva, con un diseño no experimental, transeccional y de campo. La población estuvo constituida por las universidades públicas de la Costa Oriental del Lago: Universidad del Zulia Núcleo Costa Oriental del Lago, Universidad Nacional Experimental "Rafael María Baralt", y la Universidad Politécnica Territorial del Zulia, sede Cabimas., cuyas unidades informantes comprenden tres secretarios docentes de las diferentes carreras o programas, un coordinador docente y veintisiete sujetos del personal administrativo encargado de la admisión estudiantil.

Para la recolección de datos se utilizó la encuesta a través de la aplicación de un cuestionario; el cual quedó conformado por 15 ítems con escala de frecuencia de cinco opciones de respuestas que van desde Siempre (5), Casi siempre (4), Algunas veces (3), Casi nunca (2) y Nunca (1). Se utilizó el juicio de expertos para la validez del mismo y la confiabilidad calculada por el método Alfa de Cronbach, cuyo resultando fue 0,95. El análisis de los datos se realizó mediante la estadística descriptiva, a través de la media aritmética, según el baremo diseñado por la investigadora, tal como se muestra en el cuadro 1 .

Cuadro 1. Baremo para la interpretación de la media aritmética

\begin{tabular}{cccc}
\hline Alternativas & Opción de respuesta & Rango para la media & Nivel de respuesta para la variable \\
\hline 5 & Siempre & $4.21-5.00$ & Muy alta presencia \\
4 & Casi siempre & $3.41-4.20$ & Alta presencia \\
3 & Algunas veces & $2.61-3.40$ & Moderada presencia \\
2 & Casi nunca & $1.81-2.60$ & Baja presencia \\
1 & Nunca & $1.00-1.80$ & Muy baja presencia \\
\hline
\end{tabular}

Fuente: Elaboración propia (2019) 
RESULTADOS

La tabla 1 presenta los resultados obtenidos para indicadores de eficacia que forma parte de los indicadores de gestión, en esta se aprecia un valor para la media de 3,48 indicando alta presencia. De tal forma, que estos valores certifican que en los departamentos de admisión estudiantil en las universidades analizadas, se miden los resultados de los procesos de admisión e indican por su alta presencia que se hicieron bien las actividades inherentes a dichos procesos.

Tabla 1. Indicadores de eficacia

\begin{tabular}{ccc}
\hline Indicador & $\overline{\boldsymbol{X}}$ & Categoría \\
\hline Objetivos & 3,51 & Alta presencia \\
Metas & 3,46 & Alta presencia \\
Promedio & $\mathbf{3 , 4 8}$ & Alta presencia \\
\hline
\end{tabular}

Fuente: Elaboración propia (2019)

Los resultados constatan lo expuesto por Rodríguez (2013), cuando afirma que la eficacia es el grado en que una actividad, programa $u$ organización alcanza sus metas y sus objetivos, medido a partir de sus productos (cantidad y calidad esperados, y efectos inmediatos). La eficacia demuestra la capacidad de una empresa u organización de cualquier tipo para desarrollar y mantener sistemáticamente unas ventajas comparativas que le permitan disfrutar $y$ sostener una posición destacada en el entorno socio económico en que actúa.

De igual manera, validan a la investigadora cuando afirma que, el indicador eficacia se refiere a los resultados en relación con las metas y cumplimiento de los objetivos organizacionales. Para ser eficaz se deben priorizar tareas y realizar ordenadamente aquellas que permiten alcanzarlos mejor y más rápidamente. Eficacia es el grado en que algo (procedimiento o servicio) puede lograr el mejor resultado posible.

En la tabla 2 se resume el comportamiento del indicador eficiencia, donde se observa que el mismo alcanzó un promedio de 3,48, indicando alta presencia. Según estos resultados, los departamentos de admisión estudiantil en las universidades analizadas, enfocan sus procesos en el control de los recursos o entradas; asimismo, evalúan la relación entre los recursos y su grado de aprovechamiento.

Tabla 2. Indicadores de eficiencia

\begin{tabular}{lccc}
\hline & Indicador & $\overline{\boldsymbol{X}}$ & Categoría \\
\hline Recursos humanos & & 3,59 & Alta presencia \\
Recursos materiales & 3,52 & Alta presencia \\
Recursos financieros & & 3,33 & Moderada presencia \\
& Promedio & $\mathbf{3 , 4 8}$ & Alta presencia \\
\hline
\end{tabular}

Fuente: Elaboración propia (2019) 
Estos resultados ponen de manifiesto coincidencia con lo expresado por Corredor (2007), para quien la eficiencia es la maximización de los insumos empleados para generar productos o servicios; ya sea que con recursos iguales o constantes se obtengan mayores resultados o que con resultados iguales o constantes se ejecuten menores recursos.

De manera similar, validan a la investigadora cuando señala que, la eficiencia es la capacidad administrativa de producir el máximo de resultados con el mínimo de recursos, el mínimo de energía y en el mínimo de tiempo posible; teniendo en cuenta que eficaz tiene que ver con hacer efectivo un intento o propósito. Los indicadores de eficiencia se emplean para relacionar los esfuerzos frente a los resultados que se obtengan; a mayores resultados, mayor eficiencia. Si se obtiene mejores resultados con menor gasto de recursos o menores esfuerzos, se habrá incrementado la eficiencia.

Continuando con el análisis y discusión de resultados, se muestran ahora los referidos al último indicador, denominado indicadores de efectividad. Para ello se presenta la tabla 3, donde se observa que el indicador alcanzó un promedio de 3,86 indicando alta presencia. Los hallazgos demuestran que, los departamentos de admisión estudiantil bajo estudio, miden los resultados alcanzados frente a los bienes 0 servicios generados a los clientes y usuarios.

Tabla 3. Indicadores de efectividad

\begin{tabular}{ccc}
\hline \multicolumn{1}{c}{ Indicador } & $\overline{\boldsymbol{X}}$ & Categoría \\
\hline Logro de los objetivos & 3,82 & Alta presencia \\
Satisfacción de las necesidades & 3,90 & Alta presencia \\
Promedio & $\mathbf{3 , 8 6}$ & Alta presencia \\
\hline
\end{tabular}

Fuente: Elaboración propia (2019)

Los resultados concuerdan con Jaramillo (2011), cuando afirma que, los indicadores de efectividad involucran la eficiencia y la eficacia, es decir, el logro de los resultados programados en el tiempo con los costos más razonables posibles, supone hacer lo correcto con exactitud y sin ningún desperdicio de tiempo o dinero. Asimismo, son congruentes con Rodríguez (2013), para quien la efectividad es la relación entre lo producido y la necesidad del servicio o del producto, mide en qué grado la actividad cumplida satisface la demanda del bien o servicio en términos generales.

Así las cosas, se valida el juicio de la investigadora, para quien la efectividad significa cuantificación del logro de la meta, también como la capacidad de lograr el efecto que se desea. Los indicadores de efectividad, tienen que ver con hacer realidad un intento o propósito, y están relacionados con el cumplimiento al ciento por ciento de los objetivos planteados, teniendo en cuenta que eficiencia tiene que ver con la actitud y la capacidad para llevar a cabo un trabajo o una tarea con el mínimo gasto de tiempo.

Para los indicadores de gestión, cuyos resultados se concentran en la tabla 4 , se observa un promedio de 3,61, indicando alta presencia de los de indicadores dentro de la medición de gestión que realizan en los procesos de admisión estudiantil en las universidades públicas venezolanas. 
Tabla 4. Indicadores de Gestión

\begin{tabular}{lcc}
\multicolumn{1}{c}{ Dimensión } & $\overline{\boldsymbol{X}}$ & Categoría \\
\hline Indicador de eficacia & 3,48 & Alta presencia \\
Indicador de eficiencia & 3,48 & Alta presencia \\
Indicador de efectividad & 3,86 & Alta presencia \\
\multicolumn{1}{c}{ Promedio } & $\mathbf{3 , 6 1}$ & Alta presencia \\
\hline
\end{tabular}

Fuente: Elaboración propia (2019)

Estos resultados validan los postulados de Serna (2008), cuando afirma que, se pueden clasificar los indicadores en indicadores de eficacia, eficiencia y efectividad; los cuales pueden ser utilizados para medir el desempeño de cada una de las áreas funcionales de la empresa (calidad, productividad, finanzas, mercadeo, operaciones, producción, ventas, atención al cliente, entre otros).

También alcanzan congruencia con lo expresado por la investigadora, cuando afirma que los tipos de indicadores de gestión permiten medir con parámetros enfocados a la toma de decisiones; son señales para monitorear la gestión, asegurando que las actividades vayan en el sentido correcto y permitan evaluar los resultados de una gestión frente a sus objetivos, metas y responsabilidades. Se considera importante ajustar o administrar el conjunto de indicadores a cada proceso, alineándolo con los de sus respectivas unidades de negocio y por tanto con la misión de la organización, para lograr la efectividad de los objetivos estratégicos propuestos.

\section{CONCLUSIONES}

Los resultados arrojan alta presencia de los indicadores de gestión efectividad, eficacia y eficiencia, logrando con ello que los procesos de admisión estudiantil llevados a cabo e en las universidades públicas venezolanas, puedan medirse con parámetros que enfocados a la toma de decisiones sean señales para monitorear la gestión, así se asegura que las actividades vayan en el sentido correcto y permitan evaluar los resultados de una gestión frente a sus objetivos, metas y responsabilidades.

Estos indicadores de gestión tienen como misión valorar la eficiencia de insumos, recursos y esfuerzos dedicados a obtener ciertos objetivos en un tiempo determinado y costos registrados.

\section{REFERENCIAS}

Amat, J. (2010). Control de gestión. Editorial Gestión 2000. Caracas, Venezuela

Bahamón, J. (2012) Construcción de Indicadores de Gestión bajo el Enfoque de Sistemas. Universidad ICESI. Disponible en: https://www.researchgate.net/publication/38 319754_Construccion_de_indicadores_de_ge stion_bajo_el_enfoque_de_sistemas

Barbosa, O. (2010). Los Indicadores de Gestión y su Contexto. ESAP. Bogotá

Blanco, F., Flórez, E. y Giménez, C. (2010). La equidad y la calidad en los procesos de admisión a la Educación Superior: Universidad Simón Bolívar y Universidad Central de Venezuela. Revista de Pedagogía. Versión impresa ISSN 0798-9792. Rev. Ped v.31 n.89 Caracas Venezuela

Corredor, J. (2007). La planificación estratégica. Vadell Hermanos Editores. CaracasVenezuela

Jaramillo, C. (2011). Los indicadores de gestión organizacional. Guía para su definición. Editorial LTDA. Colombia 
Pacheco, J; Castañeda, W y Caicedo, C. (2002). Indicadores integrales de gestión. McGrawHill. Colombia

Pardo, M. y Rodríguez, C. (2007). Evaluación del desempeño integral del sector transporte. Revista de investigación de la Universidad de la Salle. Volumen 7 (1). Enero - junio 2007. Bogotá

Pérez, C. (2009). Los indicadores de gestión. Disponible en: http://www.visionadministrativa.info/bibliote $\mathrm{ca} / \mathrm{control} /$ indicadores/losindicadoresdegest ion.pdf

Pérez de V. y Magro, M. (2011). Curso de habilidades directivas. Caracas: Fundación Universidad virtual Simón Rodríguez. Prentice Hall. Venezuela

Rincón, R. (2010). Los indicadores de gestión organizacional una Guía para su Definición.
Rev. Universidad EATIT111, julio, agosto, septiembre. pp. 43-58

Rodríguez, M. y Gómez, C. (2008). Indicadores de gestión para la evaluación de la eficiencia, eficacia y calidad del proceso de registro académico. Coordinación de Postgrado del DAC-UCLA

Rodríguez, R. (2013). Gestión de la calidad, Edición Díaz de Santos, S.A. Edición N ${ }^{\circ} 2$. Maestra Hefidia. España

Serna, H. (2008). Gerencia Estratégica. Teoría, Metodología. Alineamiento, Implementación y Mapas Estratégicos, Índices de Gestión. Décima edición. 3R Editores. Bogotá, Colombia 